\title{
FITORREGULADORES DE CRESCIMENTO E CAPAÇÃO NA CULTURA ALGODOEIRA $\left(^{1}\right)$
}

\author{
LUIZ HENRIQUE CARVALHO $\left({ }^{2,8}\right)$, EDERALDO JOSÉ CHIAVEGATO $\left({ }^{2,8}\right)$, \\ EDIVALDO CIA $\left({ }^{2,8}\right)$, JÚLIO ISAO KONDO $\left({ }^{3}\right)$, JOSÉ CARLOS SABINO $\left(^{4,8}\right)$, \\ ARMANDO PETTINELLI JÚNIOR( $\left(^{5}\right)$, \\ NELSON BORTOLETTO $\left({ }^{6}\right)$ e PAULO BOLLER GALLO( $\left.{ }^{7}\right)$
}

\begin{abstract}
RESUMO
Estudaram-se, em dez experimentos de campo, os efeitos dos seguintes fitorreguladores de crescimento e da capação na cultura algodoeira: cloreto de clorocolina, aplicado na dose de $50 \mathrm{~g} / \mathrm{ha}$; cloreto de chlormequat, $100 \mathrm{~g} / \mathrm{ha}$ e cloreto de mepiquat, $100 \mathrm{~g} / \mathrm{ha}$. A capação foi realizada manualmente, planta por planta, na mesma época da aplicação dos fitorreguladores, aos 60-70 dias da emergência das piantas. O delineamento estatístico foi de blocos ao acaso, com cinco tratamentos e seis repetições e, a variedade utilizada, a IAC 19. Para a avaliação dos resultados de produção e altura de plantas, efetuaram-se dois agrupamentos de ensaios, sendo um com maior e outro com menor desenvolvimento, ou seja: plantas em parcelas testemunhas com altura superior e inferior a $100 \mathrm{~cm}$ respectivamente. As demais características agronômicas e tecnológicas da fibra foram analisadas em um só grupo. Para o grupo de plantas com maior desenvolvimento, foi obtido, em média, um aumento de 16,3 e $8,4 \%$, respectivamente, para o tratamento com capação e aplicação de fitorreguladores. O fitorregulador proporcionou, em média, redução de $12,1 \%$ na altura das plantas e a capação, em média, 20,8\%. Os fitorreguladores, indistintamente, proporcionaram aumento de peso do capulho e das sementes, enquanto, com a prática da capação, não se verificou esse efeito. A aplicação de cloreto de clorocolina resultou em menor porcentagem de fibra do que o cloreto de mepiquat.
\end{abstract}

Termos de indexação: algodão, Gossypium hirsutum L., cloreto de clorocolina; cloreto de chlormequat; cloreto de mepiquat; fitorregulador de crescimento; capação; produtividade e altura de plantas.

( $\left.{ }^{1}\right)$ Trabalho apresentado na IV Reuniāo Nacional do Algodão, em Belém (PA), outubro de 1986 . Recebido para publicação em 21 de fevereiro e aceito em 14 de novembro de 1994.

$\left(^{2}\right)$ Seção de Algodão, Instituto Agronômico (IAC), Caixa Postal 28, 13001-970 Campinas (SP).

$\left({ }^{3}\right)$ Seção de Tecnologia de Fibras, IAC, Caixa Postal 28, 13001-970 Campinas (SP).

(4) Estação Experimental de Tietê, lAC, Caixa Postal 18, 18530-000 Tietê (SP).

$\left({ }^{5}\right)$ Estação Experimental de Tatuí, IAC, Caixa Postal 33, 18270-000 Tatuí (SP).

(6) Estação Experimental de Votuporanga, IAC, Caixa Postal 401, 15500-000 Votuporanga (SP).

( ${ }^{7}$ Estaçāo Experimental de Mococa, IAC, Caixa Postal 58, 13730-970 Mococa (SP).

${ }^{8}$ ) Com bolsa de pesquisa do CNPq. 


\section{ABSTRACT \\ EFFECTS OF GROWTH PHYTOREGULATORS AND PRUNING ON THE COTTON CROP}

The effects of growth phytoregulators and pruning on the cotton crop were studied in ten field experiments. The phytoregulators used were: chlorocholine chloride, applied on dose of $50 \mathrm{~g} / \mathrm{ha}$; chlormequat chloride $100 \mathrm{~g} / \mathrm{ha}$, and mepiquat chloride, applied on dose of $100 \mathrm{~g} / \mathrm{ha}$. The pruning was done handly and individually by plant, at the same period of the application of the phytoregulators by $60-70$ days after the plant emergence. The statistical delineament used was the randomized complete block design, with five treatments and six replications. The variety used was the IAC 19. Two groups of tests were done for the evaluation of yield and plant height results, one with a bigger development and another one with a smaller development, that is: plants of check plots with a height above and below $100 \mathrm{~cm}$, respectively. The others agronomic and technological fiber characteristics were analysed in an only group. For plants with the biggest development there was, on average, an increase in height of 16.3 and $8.4 \%$, respectively, for the pruning treatment and for application of the phytoregulators. The application of the phytoregulator provided, on average, a reduction of $12.1 \%$ in the height of plants. In the other hand on avcrage the practice of the pruning reduced in $20.8 \%$ the height of the plants, on average. The use of phytoregulators indistinctly, provided boll and seed weight increases, while with the practice of pruning, this effect was not significant. The application of chlormequat resulted in a lower percentage of fiber than the mepiquat chloride.

Index terms: cotton, Gossypium hirsutum L.; chlorocholine chloride; chlormequat chloride; mepiquat chloride; growth phytoregulator; pruning; productivity and plant height.

\section{INTRODUÇÃO}

A cultura do algodoeiro, no Estado de São Paulo e em outras regiões produtoras onde já se pratica uma agricultura mais tecnificada, permite o emprego econômico de fitorreguladores de crescimento visando a melhores condições de cultivo, com redução da altura das plantas e sua maior uniformização, facilitando, sobretudo, a colheita mecanizada.

O uso de fitorreguladores de crescimento em algodoeiro, principalmente quando cultivado em solos com maior fertilidade, vem aumentando no Estado de São Paulo, notadamente em propriedades onde se emprega a colheita mecanizada. Sua aplicação visa, também, substituir a capação, por vezes utilizada em pequenas propriedades agrícolas, feita manualmente, planta por planta, eliminando-se a gema apical do algodoeiro (ponteiro), para conseguir a redução do porte da planta.

No mercado brasileiro, encontram-se três produtos comerciais recomendados como fitorregula- dores de crescimento em algodoeiro: Cycocel (cloreto de clorocolina), Tuval (clore to de chlormequat) e Pix (cloreto de mepiquat).

As aplicações de cloreto de clorocolina, cloreto de chlormequat e cloreto de mepiquat, determinando redução no porte do algodoeiro, foram relatadas por diversos autores, a saber: Thomas (1964); Ferraz et al. (1977); Willard et al. (1977); Athayde (1978, 1980); Cruz et al. (1982); Yamaoka et al. (1982); Barbosa (1983); Barbosa \& Castro (1983); York (1983a,b); Cia et al. (1984); Stuart (1984); Kerby (1985); Kerby et al. (1986); Cathey \& Meredith Jr. (1988); Athayde et al. (1988); Santos et al. (1988) e Laca-Buendia (1989).

Os autores que estudaram o comportamento dos fitorreguladores de crescimento obtiveram a redução na altura das plantas, com resultados variáveis na produção de algodão em caroço. Quanto à prática da capação, existem poucos trabalhos experimentais sobre o assunto. Schmidt et al. (1962), por exemplo, demonstraram, através da análise de 
uma série de experimentos de campo em diferentes regiões paulistas qũ a capação, realizada aos 70 , 90 e 110 dias após a emergência, determinou redução na altura das plantas, sem que ocorresse aumento de produção. Laca-Buendia (1982), em Minas Gerais, estudando épocas de capação efetuada nos períodos de $50-55,60-65,70-75,80-85$ e 90-95 dias após a emergência, observou a redução média de $27 \%$ na altura das plantas em relação à testemunha apenas quando a prática foi executada entre 50 e 55 dias. Aos 60-65 dias da emergência, ocorreu um aumento de $4 \%$ na produção, comparada com a testemunha.

O objetivo deste estudo foi comparar os efeitos do emprego de vários fitorreguladores de crescimento e da capação, nas plantas de algodoeiro.

\section{MATERIAL E MÉTODOS}

Foram desenvolvidos dez experimentos, distribuídos nos municípios de Campinas, Mococa, Paranapanema e Tietê, no ano agrícola de 1982/83; de Campinas, em 1983/84, e de Campinas, Leme, Tatuí, Tietê e Votuporanga, em 1984/85.

Estudaram-se três fitorreguladores de crescimento do algodoeiro: Cycocel ou CCC, à base de cloreto de clorocolina, $50 \mathrm{~g} / \mathrm{ha}$ (tratamento 1); $\mathrm{Tu}$ val, à base de cloreto de chlormequat, $100 \mathrm{~g} / \mathrm{ha}$ (tratamento 2), e Pix, na formulação de cloreto de mepiquat, $100 \mathrm{~g} / \mathrm{ha}$ (tratamento 3). A capação (tratamento 4) foi realizada manualmente, planta por planta, na mesma época da aplicação dos fitorreguladores, aos 60-70 dias da emergência da planta. A aplicação da dose total dos fitorreguladores de crescimento do algodoeiro deu-se pela manhã, utilizando-se pulverizador de alta precisão, a 40 $\mathrm{lb} / \mathrm{pol}^{2}$, com bico X-2.

O delineamento estatístico adotado foi de blocos ao acaso, com cinco tratamentos $\mathrm{c}$ seis repetições. Cada parcela foi constituída de quatro linhas de $5 \mathrm{~m}$ de comprimento, sendo consideradas úteis as duas centrais. O espaçamento entre as linhas foi de 1,00 m, deixando-se sete plantas por metro linear após o desbaste (raleação), efetuado 25-30 dias após a emergência. Na adubação de plantio, uti- lizou-se a fórmula comercial 3-15-15, na dosagem de $400 \mathrm{~kg} / \mathrm{ha}$. Na adubação em cobertura, empregaram-se $120 \mathrm{~kg} / \mathrm{ha}$ de sulfato de amônio, logo após o desbaste. Realizaram-se capinas manuais e cultivos mecânicos, bem como aplicações de inseticidas de acordo com recomendações técnicas da Seção de Algodão do Instituto Agronômico, e utilizou-se a varicdade IAC 19.

Para avaliação dos diversos tratamentos, estudaram-se as seguintes características agronômicas: produção de algodão em caroço, porcentagem de fibra, massa $\left({ }^{9}\right)$ de cem sementes, massa de um capulho e altura média das plantas. A produção de algodão em caroço foi obtida através de duas colheitas, nas linhas centrais de cada parcela. A altura média das plantas por parcela foi determinada na primeira colheita, através da medição direta de todas as plantas existentes nas linhas úteis. Os estudos das características tecnológicas da fibra basearam-se em amostra tirada de 20 capulhos no terço médio das plantas, na primeira colheita, determinando-se: resistência intrínseca, tenacidade Pressley 1/8", micronaire, maturidade, uniformidade de comprimento e comprimento $2,5 \%$.

Efetuaram-se análises estatísticas individuais para as diferentes características estudadas em cada ensaio e análises conjuntas dos ensaios. Para produção e altura média de plantas, estabeleceram-se dois grupos em função da altura média das testemunhas: um para plantas com porte acima de $100 \mathrm{~cm}$ e outro para aquelas abaixo de $100 \mathrm{~cm}$. Foi utilizado o teste de Duncan a $5 \%$ de probabilidade, para a comparação das médias dos tratamentos.

\section{RESULTADOS E DISCUSSÃO}

Os dados médios de altura de plantas e de produção obtidos nos experimentos com menor desenvolvimento - altura média, nas parcelas testemunhas, abaixo de $100 \mathrm{~cm}$ - instalados em Paranapanema, Campinas e Leme nos anos agrícolas de $1982 / 83$ a 1984/85, encontram-se no quadro 1 .

( $\left.{ }^{9}\right)$ Massa $=$ massa determinada, em balança, em grama. 
Considerados os experimentos individualmente, verifica-se, em Campinas, que ocorreu redução no porte para tratamentos com fitorreguladores e para aquele onde se efetuou a capação. Observa-se, na média dos ensaios, que os tratamentos com fitorreguladores proporcionaram, em média, redução de $8,6 \%$ na altura das plantas em relação à testemunha, sem nunca alcançar diferença estatística. A prática de capação, por sua vez, reduziu $17,1 \%$ a altura das testemunhas, não diferindo, estatisticamente, entretanto, dos tratamentos com fitorreguladores de crescimento, a não ser em relação ao tratamento 2 . Essa tendência de redução da altura das plantas pela aplicação de fitorreguladores confirma, de certo modo, os resultados já assinalados por: Thomas (1964); Ferraz et al. (1977); Athayde (1978, 1980); Cruz et al. (1982); Yamaoka et al. (1982); Barbosa (1983); Barbosa \& Castro (1983); York (1983a,b); Cia et al. (1984); Kerby (1985); Kerby et al. (1986); Cathey \& Meredith (1988) e Laca-Buendia (1989).

Quadro 1. Médias de produção e de altura de plantas, obtidas por tratamento nos experimentos instalados em quatro locais com baixo crescimento das plantas testemunhas (menor que 100 cm), durante os anos agrícolas de 1982/83 a 1984/85

\begin{tabular}{|c|c|c|c|c|c|c|}
\hline \multirow{2}{*}{ Tratamentos } & \multirow[b]{2}{*}{ Doses } & \multicolumn{4}{|c|}{ Altura de planta $\left({ }^{1}\right)$} & \multirow{2}{*}{ Média } \\
\hline & & $\begin{array}{c}\text { Paranapanema } \\
82 / 83\end{array}$ & $\begin{array}{c}\text { Campinas } \\
83 / 84\end{array}$ & $\begin{array}{l}\text { Leme } \\
84 / 85\end{array}$ & $\begin{array}{c}\text { Campinas } \\
84 / 85\end{array}$ & \\
\hline & $\mathrm{g} / \mathrm{ha}$ & - & 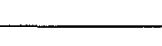 & $-\mathrm{cm}-$ & 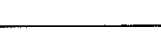 & . \\
\hline 1. Cycocel $\left({ }^{2}\right)$ & 50 & 83,5 & $87,0 \mathrm{~b}$ & $84,4 \mathrm{a}$ & $74,2 b$ & $82,3 \mathrm{ab}$ \\
\hline 2. $\operatorname{Pix}\left({ }^{2}\right)$ & 100 & 80,0 & $88,3 b$ & $87,5 \mathrm{a}$ & $81,3 \mathrm{~b}$ & $84,3 \mathrm{a}$ \\
\hline 3. Tuval $\left({ }^{2}\right)$ & 100 & 79,5 & $89,9 a b$ & $84,9 \mathrm{a}$ & $74,5 b$ & $82,2 \mathrm{ab}$ \\
\hline 4. Capação & - & 82,5 & $80,4 \mathrm{c}$ & $75,0 \mathrm{~b}$ & $62,9 c$ & $75,2 b$ \\
\hline 5. Testemunha & - & 84,5 & $95,3 \mathrm{a}$ & $87,0 \mathrm{a}$ & $96,1 \mathrm{a}$ & $90,7 \mathrm{a}$ \\
\hline Teste F & & 0,53 & $5,92 * *$ & $6,56 * *$ & $10,38 * *$ & $4,40^{*}$ \\
\hline
\end{tabular}

Produção (algodão em caroço)

\begin{tabular}{|c|c|c|c|c|c|c|}
\hline \multirow[t]{2}{*}{ Tratamentos } & \multirow[t]{2}{*}{ Doses } & Paranapanema & Campinas & Leme & Campinas & \multirow[t]{2}{*}{ Média } \\
\hline & & $82 / 83$ & $83 / 84$ & $84 / 85$ & $84 / 85$ & \\
\hline & $\mathrm{g} / \mathrm{ha}$ & \multicolumn{5}{|c|}{$-\mathrm{kg} / \mathrm{ha}$} \\
\hline 1. Cycocel $\left({ }^{2}\right)$ & 50 & 1.310 & 2.160 & 1.740 & 2.550 & 1.940 \\
\hline 2. $\operatorname{Pix} \quad\left(^{2}\right)$ & 100 & 1.490 & 2.170 & 1.940 & 2.770 & 2.090 \\
\hline 3. Tuval ( $\left.{ }^{2}\right)$ & 100 & 1.520 & 2.370 & 1.980 & 2.690 & 2.140 \\
\hline 4. Capação & - & 1.320 & 2.250 & 1.690 & 2.610 & 1.970 \\
\hline 5. Testemunha & - & 1.540 & 1.960 & 1.810 & 3.030 & 2.090 \\
\hline Teste $\mathrm{F}$ & & 0,32 & 0,85 & 1,66 & 1,02 & $1,61 \mathrm{~ns}$ \\
\hline
\end{tabular}

(1) Letras iguais indicam ausência de diferenças significativas ao nível de $5 \%$ pelo teste de Duncan. $\left({ }^{2}\right)$ Cycocel $=$ cloreto de clorocolina; Pix = cloreto de mepiquat e Tuval $=$ cloreto de chlormequat 
A eficiência da capação já havia sido demonstrada por Schmidt et al. (1962), Laca-Buendia (1982) e Beltrão et al. (1988).

Quanto à produção, verifica-se que não houve diferenças significativas, estatisticamente, entre os tratamentos estudados comparados com a testemunha. Yamaoka et al. (1982) e Cia et al. (1984) demonstraram que, quando a altura das plantas foi de aproximadamente $100 \mathrm{~cm}$, o efeito dos fitorreguladores de crescimento na produção não foi significativo. Schmidt et al. (1962), Laca-Buendia (1982) e Beltrão et al. (1988) concluíram que a prática da capação não proporcionou aumento na produção de algodão em caroço, além de requerer mais mão-de-obra.

No quadro 2, estão expostos os resultados dos experimentos com altura média das plantas testemunhas acima de $100 \mathrm{~cm}$, instalados em Tietê, Mococa e Campinas nos anos agrícolas de 1982/83 a $1984 / 85$.

Quadro 2. Médias de produção e de altura de plantas, obtidas por tratamento nos experimentos instalados em quatro locais com alto crescimento das plantas testemunhas (maior que 100 cm), durante os anos agrícolas de 1982/83 a 1984/85

\begin{tabular}{|c|c|c|c|c|c|c|}
\hline \multirow[b]{2}{*}{ Tratamentos } & \multirow[b]{2}{*}{ Doses } & \multicolumn{4}{|c|}{ Altura de planta $\left({ }^{1}\right)$} & \multirow[b]{2}{*}{ Média } \\
\hline & & $\begin{array}{l}\text { Tietê } \\
82 / 83\end{array}$ & $\begin{array}{l}\text { Mococa } \\
82 / 83\end{array}$ & $\begin{array}{c}\text { Campinas } \\
82 / 83\end{array}$ & $\begin{array}{l}\text { Tietê } \\
84 / 85\end{array}$ & \\
\hline & $\mathrm{g} / \mathrm{ha}$ & 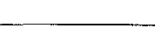 & 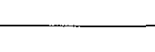 & $-\mathrm{cm}-$ & 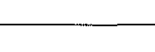 & - \\
\hline 1. Cycocel $\left({ }^{2}\right)$ & 50 & $93,0 \mathrm{~b}$ & $112,9 \mathrm{abc}$ & $96,2 b c$ & $81,0 b$ & $95,8 \mathrm{~b}$ \\
\hline 2. $\operatorname{Pix}\left({ }^{2}\right)$ & 100 & $91,2 b c$ & $110,3 \mathrm{bc}$ & $99,2 b$ & $82,0 b$ & $95,7 \mathrm{~b}$ \\
\hline 3. Tuval $\left({ }^{2}\right)$ & 100 & $88,9 \mathrm{c}$ & $115,2 \mathrm{ab}$ & $88,5 b c$ & $82,0 \mathrm{~b}$ & $93,7 \mathrm{~b}$ \\
\hline 4. Capação & - & $80,4 d$ & $102,5 \mathrm{c}$ & $87,7 \mathrm{c}$ & $70,0 \mathrm{c}$ & $85,2 \mathrm{c}$ \\
\hline 5. Testemunha & - & $111,1 \mathrm{a}$ & $122,2 \mathrm{a}$ & $111,7 \mathrm{a}$ & $106,0 \mathrm{a}$ & $112,8 \mathrm{a}$ \\
\hline Teste F & & $4,97 * *$ & $4,32 *$ & $7,63 * *$ & $17,19 * *$ & $26,6^{* *}$ \\
\hline
\end{tabular}

Produção (algodão em caroço)

Tratamentos Doses

$\begin{array}{cccc}\text { Tietê } & \text { Campinas } & \text { Votuporanga } & \text { Tietê } \\ 82 / 83 & 82 / 83 & 84 / 85 & 84 / 85\end{array}$

Média

\begin{tabular}{|c|c|c|c|c|c|c|}
\hline & & $82 / 83$ & $82 / 83$ & $84 / 85$ & $84 / 85$ & \\
\hline & $\mathrm{kg} / \mathrm{ha}$ & & & & & \\
\hline 1. Cycocel $\left({ }^{2}\right)$ & 50 & $1.770 \mathrm{~b}$ & 1.110 & $2.590 \mathrm{a}$ & 2.910 & $2.100 \mathrm{bc}$ \\
\hline 2. $\operatorname{Pix}\left({ }^{2}\right)$ & 100 & $1.870 \mathrm{ab}$ & 1.250 & $2.570 \mathrm{ab}$ & 3.340 & $2.260 \mathrm{ab}$ \\
\hline 3. Tuval $\left(^{2}\right)$ & 100 & $1.880 \mathrm{ab}$ & 1.160 & $2.710 \mathrm{a}$ & 3.210 & $2.240 \mathrm{abc}$ \\
\hline 4. Capaçăo & - & $2.180 \mathrm{a}$ & 1.040 & $2.790 \mathrm{a}$ & 3.420 & $2.360 \mathrm{a}$ \\
\hline 5. Testemunha & - & $1.810 \mathrm{ab}$ & 1.100 & $2.330 \mathrm{~b}$ & 2.870 & $2.030 \mathrm{c}$ \\
\hline Teste F & & 1,70 & 1,27 & $4,44 * *$ & 1,84 & $3,83^{*}$ \\
\hline
\end{tabular}

$\left({ }^{1}\right)$ Letras iguais indicam ausência de diferenças significativas ao nível de $5 \%$ pelo teste de Duncan. $\left({ }^{2}\right)$ Cycocel $=$ cloreto de clorocolina; $\mathrm{Pix}=$ cloreto de mepiquat $\mathrm{e}$ Tuval $=$ cloreto de chlormequat . 
Verifica-se, nas análises individuais por localidade, que houve redução na altura das plantas nos tratamentos em que se empregaram os fitorreguladores de crescimento e a capação. Na análise conjunta dos experimentos, observa-se que, nos tratamentos com fitorreguladores, ocorreu redução média da altura de $15,7 \%$ em relação à testemunha. Já a prática da capação determinou uma redução de $24,5 \%$ em relação à testemunha, diferindo, estatisticamente, desta e dos tratamentos em que se empregaram fitorreguladores.

Em relação à produção, os tratamentos que receberam os fitorreguladores de crescimento não diferiram entre si, estatisticamente, o que confirma dados obtidos por Barbosa (1983), Barbosa \& Cas- tro (1983), Cia et al. (1984) e Laca-Buendia (1989). Já o tratamento com cloreto de mepiquat diferiu estatisticamente da testemunha. A capação (tratamento 4) não diferiu dos tratamentos com cloreto de mepiquat (Pix) e cloreto de chlormequat (Tuval), mas superou estatisticamente a testemunha e o tratamento com cloreto de clorocolina (Cycocel). Em média, as análises dos diferentes ensaios para altura de plantas mostram efeitos semelhantes dos tratamentos (fitorreguladores e capação), para maior e menor desenvolvimento de plantas. Já para a produção, o efeito dos tratamentos foi mais significativo onde ocorreu maior desenvolvimento de plantas, estando de acordo com trabalhos de Schmidt et al. (1962), Yamaoka et al. (1982), Cia et al. (1984), Bcltrão et al. (1988) e Laca-Buendia (1989).

Quadro 3. Resultados médios de dez experimentos sobre a influência dos fitorreguladores de crescimento e da capação nas características agronômicas do algodoeiro e tecnológicas da fibra, nos ensaios dos anos agrícolas de 1982/83 a 1984/85

\begin{tabular}{|c|c|c|c|c|c|c|}
\hline Tratamentos & Doses & Fibra $\left({ }^{l}\right)$ & $\begin{array}{c}\text { Massa de cem } \\
\text { sementes }\left({ }^{2}\right)\end{array}$ & $\begin{array}{c}\text { Massa de um } \\
\text { capulho }\left(^{2}\right)\end{array}$ & $\begin{array}{c}\text { Resistência } \\
\text { intrínseca }\end{array}$ & Pressley 1/8" \\
\hline & $\mathrm{g} / \mathrm{ha}$ & $\%$ & $\longrightarrow$ & $g$ & $-\mathrm{g}$ & $\mathrm{ex}$ \\
\hline 1. Cycocel $\left({ }^{3}\right)$ & 50 & $38,8 \mathrm{ab}$ & $13,7 \mathrm{a}$ & $7,8 \mathrm{a}$ & 21,51 & 22,39 \\
\hline 2. $\operatorname{Pix}\left({ }^{3}\right)$ & 100 & $39,0 \mathrm{a}$ & $13,5 \mathrm{a}$ & $7,8 \mathrm{a}$ & 21,50 & 22,12 \\
\hline 3. Tuval $\left({ }^{3}\right)$ & 100 & $38,5 b$ & $13,7 \mathrm{a}$ & $7,8 \mathrm{a}$ & 21,74 & 22,33 \\
\hline 4. Capaçāo & - & $39,1 \mathrm{a}$ & $13,0 \mathrm{~b}$ & $7,5 b$ & 21,54 & 22,39 \\
\hline 5. Testemunha & - & $39,1 \mathrm{a}$ & $13,2 b$ & $7,5 b$ & 21,09 & 22,14 \\
\hline Teste F & & $6,33 * *$ & $13,77 * *$ & $6,16^{* *}$ & 2,16 & 1,64 \\
\hline
\end{tabular}

Tratamentos Doses $\quad$ Micronaire $\quad$ Maturidade $\begin{gathered}\text { Uniformidade } \\ \text { de comprimento }\end{gathered}$ Comprimento $2.5 \%$

\begin{tabular}{lccccc} 
& $\mathrm{g} / \mathrm{ha}$ & & & $\mathrm{mm}$ \\
1. Cycocel $\left({ }^{3}\right)$ & 50 & 4,4 & 58,10 & 46,22 & 27,67 \\
2. Pix $\left(^{3}\right)$ & 100 & 4,5 & 58,11 & 46,05 & 27,60 \\
3. Tuval $\left(^{3}\right)$ & 100 & 4,4 & 58,32 & 45,96 & 27,53 \\
4. Capação & - & 4,4 & 57,50 & 45,84 & 27,52 \\
5. Testemunha & - & 4,4 & 57,27 & 46,04 & 27,57 \\
Teste F & & 1,72 & 1,09 & 1,23 & 0,86 \\
\hline
\end{tabular}

( $\left.{ }^{1}\right)$ Letras iguais indicam ausência de diferenças significativas ao nível de $5 \%$ pelo teste de Duncan. $\left({ }^{2}\right)$ Massa determinada em balança $(\mathrm{g}) .\left({ }^{3}\right)$ Cycocel $=$ cloreto de clorocolina; $\mathrm{Pix}=$ cloreto de mepiquat e Tuval = cloreto de chlormequat. 
Quanto ao estudo das demais características agronômicas e tecnológicas da fibra - Quadro 3 - verifica-se que, na média, os fitorreguladores de crescimento aumentaram a massa do capulho e das sementes em relação à testemunha e ao tratamento com capação. O tratamento com Tuval apresentou redução para porcentagem de fibra em relação ao Pix, capação e testemunha. Esses dados confirmam aqueles obtidos em trabalhos de autores citados.

Para a maioria das características tecnológicas da fibra, não ocorreram diferenças significativas entre os vários tratamentos.

\section{REFERÊNCIAS BIBLIOGRÁFICAS}

ATHAYDE, M.L.F. Efeito do CCC no algodoeiro (Gossypium hirsutum L.). Piracicaba, 1978. 51p. Tese (Mestrado) - ESALQ/USP, 1978.

ATHAYDE, M.L.F. Efeitos de N e cloreto de clorocolina (CCC) no metabolismo nitrogenado e em algumas características do algodoeiro (Gossypium hirsutum L.). Piracicaba, 1980. 94p. Tese (Doutorado) ESALQ/USP, 1980.

ATHAYDE, M.L.F.; SOUZA JÚNIOR., J.E.; PARDUCCI, S. \& PIPOLO, A.E. Efeito do volume de calda e doses de CCC aplicados em algodoeiro, na fase inicial e média do florescimento. In: REUNIÃO NACIONAL DO ALGODÃO, 5., Campina Grande, 1988. Resumos. Campina Grande, 1988. p.78.

BARBOSA, L.M. Efeitos de reguladores vegetais em algodoeiro (Gossypium hirsutum L. cv. IAC 17). Piracicaba, 1983. 97p. Tese (Mestrado) - ESALQ/USP, 1983.

BARBOSA, L.M. \& CASTRO, P.R.C. Desenvolvimento e produtividade de algodoeiros sob efeito de reguladores vegetais. Anais da Escola Superior de Agricultura "Luiz de Queiroz", Piracicaba, XL:33$86,1983$.

BELTRÃO, N.E. DE M.; DINIZ, M. DE S.; VIEIRA, D.J.; NÓBREGA, L.B. da; SOUSA, R.P. de \& SOUSA NETO, J.B. de. Configuração de plantio e época de capação em algodoeiro herbáceo de curta duração irrigado. In: REUNIÃO NACIONAL DO ALGODÃO, 5., Campina Grande, 1988. Resumos. Campina Grande, 1988. p.70.
CATHEY, G.W. \& MEREDITH JR., W.R. Cotton response to planting date and mepiquat chloride. Agronomy Journal, Madison, 80:463-466, 1988.

CIA, E.; CARVALHO, L.H.; KONDO, J.I.; FUZATTO, M.G.; BORTOLETTO, N.; GALLO, P.B.; CRUZ, L.S.P.; SABINO, N.P.; PETTINELLI JÚNIOR., A.; MARTINS, A.L.M. \& SILVEIRA, J.C.O. Efeito de clorocolina e cloreto de mepiquat na cultura do algodoeiro. Planta Daninha, Campinas, 7(2):23-36, 1984.

CRUZ, L.S.P.; SABINO, N.P. \& TOLEDO, N.M.P. Efeitos do cloreto de mepiquat empregado como fitorregulador sobre algodoeiro herbáceo (Gossypium hirsutum L. IAC 16). Planta Daninha, Campinas 5(1):15-18, 1982.

FERRAZ, C.A.M.; CIA, E.; SABINO, N.P.; GROSSI, J.M.M.; VIEGA, A.A. \& YOSHIDA, H. Efeitos da densidade de plantio e da aplicação de $\mathrm{CCC}$ em algodoeiro. Bragantia, Campinas, 36:39-51, 1977.

KERBY, T.A. Cotton response to mepiquat chloride. Agronomy Journal, Madison, 77:515-518, 1985.

KERBY, T.A.; HAKE, K. \& KEELEY, M. Cotton fruiting modification with mepiquat chloride. Agronomy Journal, Madison, 78:907-912, 1986.

LACA-BUENDIA, J.P. DEL C. Efeito da época de capação na produtividade do algodoeiro herbáceo (Gossypium hirsutum L.) no Triângulo Mineiro. Campina Grande, EMBRAPA-CNPA, 1982. $11 \mathrm{p}$. Circular Técnica, 5)

LACA-BUENDIA, J.P. DEL C. Efeito de doses de reguladores de crescimento no algodoeiro (Gossypium hirsutum L.). Revista Brasileira de Fisiologia Vegetal, Londrina, 1(1):109-113, 1989.

SANTOS, E. de A.; BARROSO, A.L. de L. \& PRADO, P.C.N. do. Efeito de fitorreguladores de crescimento em duas variedades de algodoeiro de porte e ciclos diferentes. In: REUNIÃO NACIONAL DO ALGODÃO, 5., Campina Grande, 1988. Resumos. Campina Grande, 1988. p.64.

SCHMIDT, W.; AGUIAR, H.C. de \& CORREAA, D.M. Ensaios sobre capação e desbrota do algodoeiro. Bragantia, Campinas, 21:425-448, 1962.

STUART, B.L.; ISBELL, V.R.; WENDT, C.W. \& ABERNATY, J.R. Modification of cotton water relations and growth with mepiquat chloride. Agronomy Journal, Madison, 76:651-655, 1984. 
THOMAS, O.R. Effects of application timing and concentration of 2 chloroethyl trimethylammonium chloride on plant size and fruiting response of cotton. Crop. Science, Madison, 4:403-405, 1964.

WILLARD, J.J.; KUPELIAN, R.H. \& SCHOTT, P.E. Effect of 1,1 Dimethyl piperidinium chloride on cotton yield and development. In: BELTWIDE COTTON PRODUCTION RESEARCH CONFERENCE, Atlanta, 1977. Proceedings, Atlanta, 1977. p.69.
YAMAOKA, R.S.; PIRES, J.R. \& ALMEIDA, W.P. de. Estudo de época de parcelamento de aplicação de fitohormônio em diferentes populações de plantas. In: REUNIÃO NACIONAL DO ALGODÃO, 2., Salvador, 1982. Resumos. Salvador, EMBRAPA, 1982. p.110.

YORK, A.C. Cotton cultivar response to mepiquat chloride. Agronomy Journal, Madison, 75:663-666, $1983 \mathrm{a}$.

YORK, A.C. Response of cotton to mepiquat chloride with varying $\mathrm{N}$ rates and plant populations. Agronomy Journal, Madison, 75:667-672, 1983b. 